\title{
Existence of the Scattering Matrix for the Linearized Boltzmann Equation
}

\author{
Barry Simon $\star$ \\ Departments of Mathematics and Physics, Princeton University, Princeton, New Jersey, USA
}

Received October 15, 1974

\begin{abstract}
Following Hejtmanek, we consider neutrons in infinite space obeying a linearized Boltzmann equation describing their interaction with matter in some compact set $D$. We prove existence of the $S$-matrix and subcriticality of the dynamics in the (weak-coupling) case where the mean free path is larger than the diameter of $D$ uniform in the velocity. We prove existence of the $S$-matrix also for the case where $D$ is convex and filled with uniformly absorbent material. In an appendix, we present an explicit example where the dynamics is not invertible on $L_{+}^{1}$, the cone of positive elements in $L^{1}$.
\end{abstract}

\section{§1. Introduction}

In this paper, we consider the linearized Boltzmann equation (LBE):

$$
\dot{n}(x, v, t)=-v \cdot \operatorname{grad}_{x} n(x, v, t)+\int k\left(x, v^{\prime}, v\right) n\left(x, v^{\prime}, t\right) d v^{\prime}-\sigma_{a}(x, v) n(x, v, t) .
$$

This equation describes a beam of neutrons which is non-self-interacting; thus we are assuming low density and the non-linear term is dropped from the usual Boltzmann equation (3.6). The first term describes free streaming of the beam in phase space, the second the net input in $(x, v)$ phase space due to scattering from other regions $\left(x, v^{\prime}\right)$ in phase space and from production (fission!) by other particles. Simiarly, the last term describes loss due to absorbtion and scattering from $(x, v)$ into other regions. We emphasize that while we use the symbol $\sigma_{a}$ (in order to have notation similar to Hejtmanek [7]), $\sigma_{a}$ is not quite a cross-section but has the units of inverse time and is a rate. $v^{-1} \sigma_{a}(x, v)$ is the cross-section times the density of scatters (or absorbers) and is an inverse mean free path. Similarly the quantity

$$
\sigma_{p}(x, v)=\int k\left(x, v, v^{\prime}\right) d v^{\prime}
$$

is a production rate [note that $k\left(x, v, v^{\prime}\right)$ appears in (2) but that $k\left(x, v^{\prime}, v\right)$ appears in (1)!].

In the cases of greatest physical interest $[1,3,5,16]$, either the configuration space is finite or it is made effectively finite by having pure absorbers $(k=0$; $\left.\sigma_{a}(x, v) \geqq \alpha\right)$ arround an interaction region $D$ (so the beam delays exponentially outside $D$ ). In some approximations $[9,14,15] D$ is taken to be a slab infinite in two directions. In any event, the case we will consider (following Hejtmanek [7]) of free space surrounding a interaction region is quite far from reactor theory. It is nevertheless of some physical interest and is of special interest in the mathematical theory of scattering $[10,12,19]$ for two reasons: first the natural data set

* A. Sloan fellow; research partially supported by the U.S. NSF under Grant GP 39048 
for the problem is not a Hilbert space; in fact it is not even a vector space, for it is $L_{+}^{1}\left(\mathbb{R}^{m}\right)$, the cone of positive functions in $L^{1}\left(\mathbb{R}^{m}\right)$. Of course, for technical purposes the vector space $L^{1}$ is useful. Secondly, the interacting dynamics, $W(t)$ is, in general only one sided, i.e. defined from $L_{+}^{1}$ to $L_{+}^{1}$ only for $t \geqq 0$. This phenomena occurs even in the pure scattering case where $\sigma_{a}(x, v)=\sigma_{p}(x, v)$ (see the Appendix) and is thus not due to energy or number dispension but rather due to the statistical nature of the Boltzmann equation as expressed, for example, in the celebrated $H$-theorem. (To confuse the issue $W(t)$ is invertible on $L^{1}$ but with an inverse that can be non-positivity preservity.) For one-sided dynamics, the basic objects of scattering theory are [13]:

$$
\begin{aligned}
& \Omega^{+}=\underset{t \rightarrow-\infty}{s-\lim _{t \rightarrow-}} W(-t) W_{0}(t), \\
& \tilde{\Omega}^{-}=\underset{t \rightarrow+\infty}{s-\lim _{t \rightarrow+\infty}} W_{0}(-t) W(t)
\end{aligned}
$$

and the $S$-matrix $S=\tilde{\Omega}^{-} \Omega^{+}$. Notice that the free dynamics $W_{0}(s)$ occurs in (3) for $s \leqq 0$ and so must be two sided but that only $W(s), s>0$ occurs.

The structure of this paper is the following. In $\S 2$, we present the basic general elements of the theory reviewing the work of Hejtmanek [7] whose two main theorems concern the soluability of the Cauchy problem for $t>0$ and the existence of the limit (3a) in the case of absorption or pure scattering i.e., $\sigma_{p}(x, \sigma) \leqq \sigma_{a}(x, \sigma)$. We review this work partly for the readers convience, partly to establish notation, partly because we need lemmas in a form slightly different from this and partly to include some trivial extensions, most notably the existence of $\Omega^{+}$in the subcritical case $\sup _{t \geqq 0}\|W(t)\|<\infty$. In $\S 3$, we discuss the weak coupling case (physically the case of mean free path larger than the interaction regions) following Kato's discussion [11] of weakly coupled two-body Schrödinger systems. Our main results assert that in this case the system is subcritical and the limit $(3 \mathrm{~b})$ exists. In $\S 4$, we consider the strictly absorbing case, $\sigma_{a}(x, v)-\sigma_{p}(x, v) \geqq \alpha>0$ all $x \in D$, the interaction region and show that when $D$ is convex, the limit ( 3 b) exists. In an appendix we give an example where $W(t)$ is not invertible as a map from $L_{+}^{1}$ to $L_{+}^{1}$.

Acknowledgements. It is a pleasure to thank T. Hejtmanek for discussions and communication of [7] before publication and R. Werner and C. Wilcox for an invitation to the Oberwolfach Scattering Theory Conference where I learned of [7] and this work was begun.

\section{§2. Foundations of the Theory (Following-Hejtmanek)}

In what follows, we study Eq. (1) where $\sigma_{a}(x, v)$ and $k\left(x, v^{\prime}, v\right)$ are given measurable functions respectively on $\mathbb{R}^{2 n}$ and $\mathbb{R}^{3 n}$. They must be a.e. non-negative and obey:

(CS) $\sigma_{a}$ and $K$ have compact support in $x$-space, i.e. there is a compact $D$, with $\sigma_{a}(x,$.$) and K(x, .$.$) identically zero for x \notin D$. then

(FR) There are uniform bounds on the reaction rates, i.e. if $\sigma_{r}$ is given by (2),

$$
R(\sigma) \equiv \sup _{x, v \in \mathbb{R}^{6}}\left[\sigma_{r}(x, v)+\sigma_{a}(x, v)\right]<\infty
$$


Occasionally, we will require:

(FM) There are uniform lower bounds on the mean free paths, i.e.

$$
M(\sigma) \equiv \sup _{x, v \in \mathbb{R}^{6}}\left[|v|^{-1}\left(\sigma_{p}(x, v)+\sigma_{a}(x, v)\right)\right]<\infty .
$$

All these conditions are quite reasonable physically. $R_{a}(\sigma), R_{r}(\sigma), M_{a}(\sigma), M_{r}(\sigma)$ will denote the obvious terms. In the LBE, Eq. (1), the two operators:

and

$$
\left(A_{1} n\right)(x, v)=-\int k\left(x, v^{\prime}, v\right) n\left(x, v^{\prime}\right) d v^{\prime}
$$

$$
\left(A_{2} n\right)(x, v)=\sigma_{a}(x, v) n(x, v)
$$

enter. We have:

Lemma 2.1. If (FR) holds then $A_{1}$ and $A_{2}$ define bounded operators on $L^{1}\left(\mathbb{R}^{2 n}\right)$ with

$$
\left\|A_{1}\right\| \leqq R_{r}(\sigma) ; \quad\left\|A_{2}\right\| \leqq R_{a}(\sigma) .
$$

The semigroups $e^{-t A_{1}}(t \geqq 0, i=1,2)$ are positivity preserving i.e. they leave the cone $L_{+}^{1}$ invariant. If (FM) holds, then in addition $A_{1}|v|^{-1}$ and $A_{2}|v|^{-1}$ are bounded where $|v|^{-1}$ is the operator taking $n(x, v)$ to $|v|^{-1} n(x, v)$ and

$$
\left\|A_{1}|v|^{-1}\right\| \leqq M_{r}(\sigma) ; \quad\left\|A_{2}|v|^{-1}\right\| \leqq M_{a}(\sigma) .
$$

Proof. The boundedness statements for $A_{2}$ are trivial and those for $A_{1}$ are simple consequences of Fubini's theorem. Since $e^{-t A_{2}}$ is multiplication by $e^{-t \sigma_{a}}$ it is clearly positivity preserving. Since $-A_{1}$ is positivity preserving so is

$$
e^{-t A_{1}}=\sum_{n=0}^{\infty} t^{n} \frac{\left(-A_{1}\right)^{n}}{n !} .
$$

We also need the operator $T_{0}$ which is the closure of the operator defined on $C_{0}^{\infty}\left(\mathbb{R}^{2 n}\right)$ by:

$$
\left(T_{0} n\right)(x, v)=\left(v \cdot \operatorname{grad}_{x} n\right)(x, v) .
$$

Lemma 2.2. $T_{0}$ is the generator of a one parameter group of positivity preserving isometries on $L^{1}\left(\mathbb{R}^{2 n}\right)$ and $W_{0}(t)=e^{-t T_{0}}$ is given by:

$$
\left(W_{0}(t) n\right)(x, v)=n(x-v t, v) .
$$

Proof. The group given by (6) clearly has the claimed properties so we must only show that $T_{0}$ is its generator. For $n \in C_{0}^{\infty}\left(\mathbb{R}^{2 n}\right)$ it is clear that

$$
\left.\frac{-d}{d t} W_{0}(t) n\right|_{t=0}=T_{0} n
$$

so the generator clearly extends $T_{0}$. We are thus reduced to showing that $C_{0}^{\infty}\left(\mathbb{R}^{2 n}\right)$ is a core for the generator. But since $C_{0}^{\infty}$ is left invariant by $W_{0}(t)$ this follows from a theorem of Nelson [17] (see e.g. Theorem $X$ of [18]).

We can now easily solve the Cauchy problem for the (LBE), Eq. (1):

Theorem 2.3 (essentially in Hejtmanek [7]). If (FR) holds, then the closure of $T=T_{0}+A_{1}+A_{2}$ defined on $C_{0}^{\infty}\left(\mathbb{R}^{n}\right)\left[D(T)=D\left(T_{0}\right)\right]$ is the generator of a one 
parameter strongly continuous semigroup $W(t)=e^{-t T}$ on $L^{1}$ leaving $L_{+}^{1}$ invariant with

$$
\|W(t)\| \leqq \exp \left(t R_{r}(\sigma)\right) .
$$

Proof. That $T$ generates an exponentially bounded strongly continuous semigroup is a standard perturbation result of Phillips (see [8] or [18]). Since each of the semigroups $e^{-t A_{i}}, W_{0}(t)$ are positivity preserving and since

$$
\left\|e^{-t} A_{i}\right\| \leqq \exp \left(t R_{r}(\sigma)\right), \quad\left\|e^{-t A_{2}}\right\| \leqq 1, W_{0}(t) \leqq 1,
$$

the bound (7) and the fact that $e^{-T t}: L_{+}^{1} \rightarrow L_{+}^{1}$ follow from the Trotter product formula $[2,20]$.

Remark. While $e^{+t T}$ exists, it may not take $L_{+}^{1}$ to $L_{+}^{1}$; see the appendix.

For latter purposes we note two formulae from the perturbation theory of semigroups [8] which we will need later. Let $A=A_{1}+A_{2}$. Then the Du Hamel expansion says:

$$
W(t)=W_{0}(t)-\int_{0}^{t} W_{0}(t-s) A W(s) d s
$$

we will also need its convergent iteration, the Dyson-Phillips expansion:

$$
W(t)=\sum_{n=0}^{\infty}(-1)^{n} \int_{\substack{s_{1} \geqq 0 \ldots s_{n} \geqq 0 \\ s_{1}+\cdots+s_{n} \leqq t}} W_{0}\left(t-s_{1} \ldots s_{n}\right) A W_{0}\left(s_{1}\right) \ldots A W_{0}\left(s_{n}\right) d^{n} s .
$$

For there to be a sensible scattering theory, we need to be in the case where the material of which we are scattering can be neither a reactor nor a bomb:

Definition. We say the system $\left(k, \sigma_{a}\right)$ is subcritical if

$$
\sup _{t \geqq 0}\|W(t)\|<\infty
$$

It is physically obvious that:

Theorem 2.4 (essentially in Hejtmanek [7]).

(a) If $\sigma_{p}(x, v) \leqq \sigma_{a}(x, v)$, then $\|W(t)\| \leqq 1(t \geqq 0)$ and in particular the system is subcritical.

(b) If $\sigma_{p}(x, v)=\sigma_{a}(x, v)$ (pure scattering) then $W(t)$ is an isometry on $L_{+}^{1}$.

Remark. If $W(t)$ is not invertible on $L_{+}^{1}$ it will not be an isometry on $L^{1}$ in case (b).

Proof. Since $|W(t) f| \leqq W(t)|f|$ on account of the fact that $W$ is positivity preserving, we have only to prove $\|W(t) n\| \leqq\|n\|$ (resp. $=\|n\|)$ in case (a) [case (b)] for $n \geqq 0$. Since $W_{0}$ is an isometry, the Trotter product formula implies we need only show that $\left\|e^{-t A} n\right\| \leqq\|n\|(=\|n\|)$ in case (a) [resp. (b)]. But

$$
\int(-A n)(x, v) d x d v=\int\left[\sigma_{p}(x, v)-\sigma_{a}(x, v)\right] n(x, v) d x d v
$$

which is $\leqq 0,(=0)$ in case (a) [resp. (b)] and thus $e^{-t A}$ decreases $\int n(x, v) d x d t$. 
In $\S 3$, we will show that certain systems violating $\sigma_{p} \leqq \sigma_{a}$ are subcritical.

Hejtmanek's final theorem asserts the existence of $\Omega^{+}$in the cases covered by Theorem 2.4. For later purposes, we will need a lemma which implies the existence of $\Omega^{+}$although by a proof less "physical" than Hejtmanek's:

Lemma 2.5. For any Borel set $D$, let $\|n\|_{D} \equiv \int_{x \in D} n(x, v) d x d v$.

Then for $n \in L^{1}$

$$
\int_{-\infty}^{\infty}\left\|W_{0}(t) n\right\|_{D} d t \leqq(\operatorname{diam} D)\left\||v|^{-1} n\right\|
$$

where $\operatorname{diam} D=\sup _{x, y \in D}|x-y|$.

Proof. We will show that for each fixed $v$ :

$$
\int_{x \in D} \int_{-\infty}^{\infty}\left|\left(W_{0}(t) n\right)(x, v)\right| d x d t \leqq \operatorname{diam} D \int|v|^{-1}|n(x, v)| d x
$$

whence (10) follows by integretating over $v$ and using Fubini's theorem. Letting $\mathscr{X}$ be the characteristic function of $D$, the left hand side of (11) is

$$
\int_{-\infty}^{\infty} \int \mathscr{X}(x)|n(x-v t, v)| d x d t
$$

Let $y$ be the coordinate in $x$-space parallel to $v$ and $x_{\perp}$ the orthogonal coordinates. Then this last integral is

$$
\int_{-\infty}^{\infty} \int d t \int \mathscr{X}\left(y, x_{\perp}\right)\left|n\left(y-|v| t, x_{\perp}, v\right)\right| d y d x_{\perp} d t
$$

letting $z=y-v t$ and changing variables from $y, t$ to $y, z$ we see that the integral is

$$
\left.\left[|v|^{-1} \int\left[\int \mathscr{X}\left(y, x_{\perp}\right) d y\right]\left[\int \mid n\left(z, x_{\perp}, v\right)\right] d z\right] d x_{\perp}\right]
$$

Thus (11) follows form the bound

$$
\left(\int \mathscr{X}\left(y, x_{\perp}\right) d y\right) \leqq \operatorname{diam} D
$$

uniform in $x_{\perp}$ and this bound is geometically clear.

Theorem 2.6 (essentially in Hejtmanek [7]). If $\langle k, \sigma\rangle$ obey (CS), (FR) and define a subcritical system, then the limit:

$$
\Omega^{+}=\underset{t \rightarrow-\infty}{s-\lim _{t \rightarrow \infty}} W(-t) W_{0}(t)
$$

exists. $\Omega^{+}$is positively preserving, is a contraction if $\sigma_{p} \leqq \sigma_{a}$ pointwise and an isometry if $\sigma_{p}=\sigma_{a}$ pointwise.

Proof. We use Cook's method [4]. Since the $\left\|W(-t) W_{0}(t)\right\|$ are uniformly bounded, we need only prove the limit exists for a dense set of $n \in L^{1}$. The prop- 
erties of $\Omega^{+}$then follows from the properties we have proven above for $W$ and $W_{0}$. Now, it follows from standard perturbation theory that for $s<t$

$$
\begin{aligned}
\| W(-t) W_{0}(t) n & -W(-s) W_{0}(s) n\|=\| \int_{s}^{t} W(-u) A W_{0}(u) n d u \| \\
& \leqq \int_{s}^{t}\left\|W(-u) A \mathscr{X}_{D} W_{0}(u) n d u\right\| \\
& \leqq\left(\sup _{w \geqq 0}\|W(w)\|\right)\|A\| \int_{s}^{t}\left\|W_{0}(u) n\right\|_{D} d u
\end{aligned}
$$

where we have used the fact that by (CS), $A n$ only depends on the values of $n$ in $\mathscr{Z}_{D}$. If $|v|^{-1} n \in L^{1}, \int_{-\infty}^{<\infty}\left\|W_{0}(u) n\right\|_{D} d u<\infty$ by Lemma 2.5 and thus

$$
\lim _{t, s \rightarrow-\infty} \int_{s}^{t}\left\|W_{0}(u) n\right\|_{D} d u \rightarrow 0 \quad \text { so } \quad W(-t) W_{0}(t) n
$$

is Cauchy.

There is clearly a close connection between subcritility and the existence of $\tilde{\Omega}^{-}$since both are expressions of the fact that eventually the norm $\|n\|_{D}$ goes to zero. In fact we will make:

Conjecture $\tilde{\Omega}^{-}$exists for any subcritical system.

\section{§3. Weakly Coupled Systems}

Our analysis depends on the following basic result:

Theorem 3.1. If $\left(L^{1}\right)_{+}$contains a dense set for which

$$
\int_{0}^{\infty}\|W(t) n\|_{D} d t<\infty
$$

then under hypotheses (CS), (FR) and subcriticality the limit

$$
\tilde{\Omega}^{-}=\underset{t \rightarrow+\infty}{s-\lim } W_{0}(-t) W(t)
$$

exists. If

$$
\int_{0}^{\infty}\|W(t) n\|_{D} d t \leqq C\|n\|
$$

then the system is subcritical.

Proof. As in the proof of Theorem 2.6,

$$
\left\|\left[W_{0}(-t) W(t)-W_{0}(-s) W(s)\right] n\right\| \leqq\|A\| \int_{s}^{t}\|W(u) n\|_{D} d u
$$

so that (12) implies the limit $\tilde{\Omega}^{-} n$ exists for a dense set of $n$. Subcriticality implies the necessary bound to extend the convergence to all $n$. 
Let (13) hold; then using (8), we see that

$$
\begin{aligned}
\|W(t) n\| & \leqq\|n\|+\int_{0}^{t}\|A W(s) n\| d s \\
& \leqq\|n\|+\|A\| \int_{0}^{t}\|W(s) n\|_{D} d s \leqq(1+\|A\| C)\|n\|
\end{aligned}
$$

implying subcriticality.

Our basic small coupling result is patterned after the time-dependent aspects of Kato's theory of smooth perturbations [11]:

Theorem 3.2. If $(k, \sigma)$ obey $(C S),(F R)$ and $(F M)$ and if $\alpha=(\operatorname{diam} D) M(\sigma) \leqq 1$, then for any $n$ :

$$
\int_{0}^{\infty}\|A W(t) n\| d t \leqq \alpha(1-\alpha)^{-1}\|n\| .
$$

The wave operator $\tilde{\Omega}^{-}$exists and the system is subcritical.

Proof. We will first show that for any $n \in L^{1}$

$$
\int_{0}^{\infty}\left\|A W_{0}(t) n\right\| d t \leqq \alpha\|n\|
$$

For $\left\|A v^{-1}\right\| \leqq M(\sigma)$ by Lemma 2.1 and since $v$ commutes with $W_{0}$ :

$$
\begin{aligned}
\int_{0}^{\infty}\left\|v W_{0}(t) n\right\|_{D} d t & \leqq \int_{-\infty}^{\infty}\left\|W_{0}(t) v n\right\|_{D} d t \\
& \leqq(\operatorname{diam} D)\|n\|
\end{aligned}
$$

by Lemma 2.5. Since $\left\|A W_{0}(t) n\right\| \leqq\left\|A v^{-1}\right\|\left\|v W_{0}(t) n\right\|_{D}$, (15) follows. (15) allows us to bound the terms (9):

$$
\begin{aligned}
\int_{0}^{\infty}\|A W(t) n\| d t & \leqq \sum_{n=1}^{\infty} \int\left\|A W_{0}\left(s_{1}\right) \ldots A W_{0}\left(s_{2}\right) \ldots A W_{0}\left(s_{n}\right) n\right\| d^{n} s \\
& \leqq\left(\alpha+\alpha^{2}+\cdots\right)\|n\|=\alpha(1-\alpha)^{-1}\|n\| .
\end{aligned}
$$

This proves (14) and from (14) we can conclude the subcritibility and existence of $\tilde{\Omega}^{-}$by minor changes in the proof of Theorem 5.1 .

Remarks. 1. Theorem 5.2 has as a corollary the existence of a critical diameter for critical or supercritical behavior. It is a little disappointing that the result does not have a critical volume (mass) instead but it seems difficult for this to happen without additional assumptions on $\sigma, k$.

2. The condition $M(\sigma)^{-1} \geqq(\operatorname{diam} D)$ is essentially a statement that the mean free path in larger than $D$ and is physically quite satisfying.

\section{§4. Uniformly Absorbing Material}

We say the region $D$ is uniformly absorbing, if for all $x \in D, v \in \mathbb{R}^{n}, \sigma_{a}(x, v)$ $-\sigma_{p}(x, v) \geqq \alpha$. Of course, in this case, $W(t)$ is subcritical by Theorem 2.4. What we wish to prove is:

Theorem 4.1. If $D$ is uniformly absorbing and convex, then the inverse wave operator $\tilde{\Omega}^{-}$exist. 
The intuition behind Theorem 4.1 is that particles inside $D$ either leave $D$ in which case they should never return, or they stay in $D$ in which case they are absorbed expontially. We thus expect that for any "nice" $n,\|W(t) n\|_{D} \leqq C e^{-\alpha t}$ so that (12) holds. We will first prove a lemma that says that for large enough times nothing now can enter $D$ (first half of the above argument).

Lemma 4.2. Let $D \subset S_{r}$, the open ball of radius $r$. For each $x \in S_{r} \backslash D$, let $C_{x}=$ set of $v \in \mathbb{R}^{n}$ such that the half ray $x$-vt intersects $D$. Let

$$
n \in \hat{C}_{0}^{\infty}\left(R^{2 n}\right)=\left\{n \in C_{0}^{\infty}\left(R^{2 n}\right) \mid \operatorname{supp} n \cap\{\langle x, v\rangle \mid v=0\}=\phi\right\} .
$$

Then for sufficiently large $t, n(x, v, t) \equiv(W(t) n)(x, v)=0$ if $x \in S_{r} \backslash D, v \notin C_{x}$.

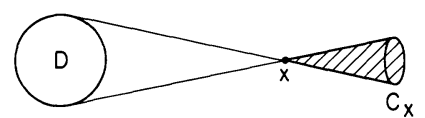

Fig. 1 The Cone $C_{x}$

Proof. By (8):

$$
n(t)=W_{0}(t) n-\int_{0}^{t} W_{0}(s) A n(t-s) d s .
$$

For $n \in \hat{C}_{0}^{\infty}$, we can clearly choose $T$ so that for $t>T, x \in S_{r}\left(W_{0}(t) n\right)(x, v)=0$ all $v$; for $\operatorname{supp}_{x}\left(W_{0}(t) n\right) \rightarrow \infty$. Thus $x \in S_{r}, n(x, v, t) \neq 0$ implies that $W_{0}(s)(A n)$ $\cdot(x, v, t-s) \neq 0$ for some $s>0$ which implies $A n(x-v s, v, t-s) \neq 0$ for some $s$ which implies that $x-v s \in D$ for some $s>0$. Thus $v \in C_{x}$.

Next we need a simple geometric fact. Given $D$, let $p(x)=\operatorname{dist}(x, D)$. Then:

Lemma 4.3. If $D$ is convex, then for any $x \in S_{r} \backslash D$ and $v \in C_{x}, v \cdot \operatorname{grad} p \geqq 0$.

Proof. Given $x \in S_{r} \backslash D$, let $y$ be the point in $D$ with $\operatorname{dist}(y, x)=p(x)$. Such a $y$ exists by compactness and is unique by convexity and the parallelogram law. Let $\hat{e}$ be the unit vector in direction $y-x$, and let $P$ be the plane through $x$ orthogonal to $\hat{e}$. By definition of $y$, if $z \in D$, then the angle $\alpha=x y z$ must be larger than $90^{\circ}$ so that $x z$ must point in an accute direction relative to $\hat{e}$, i.e. $C_{x}$ lies on the opposite side of $C_{x}$ from $\hat{e}$ (Fig. 2). If we show that $\operatorname{grad} p=-\hat{e}$ we will be done. But this follows from convexity.

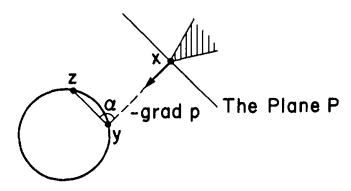

Fig. 2

Theorem 4.4. Under the hypothesis of Theorem 4.1, for any $n \in \hat{C}_{0}^{\infty}$, there is a $T$ with $\|n(t)\|_{D} \leqq e^{-\alpha(t-T)}\|n(T)\|_{D}$ for $t \geqq T$. 
Proof. Let $\phi$ be a monotone decreasing $C_{0}^{\infty}$ function on $[0, \infty]$ with $\phi(0)=1$ and with $\operatorname{supp} \phi(p(x)) \subset S_{r}$ some fixed open ball containing $D$. Let

$$
\|n\|_{\phi}=\int \phi(p(x)) n(x, v) d x d v
$$

for $n \in L_{+}^{1}$. Then by Eq. (1):

$$
\frac{d}{d t}\|n\|_{\phi}=\int\left(v \cdot \operatorname{grad}_{x}\right) \phi(p(x)) n(x, v) d x d v+\text { remainder }
$$

where the remainder obeys:

$$
\begin{aligned}
\text { remainder } & =\int k\left(x, v^{\prime}, v\right) n\left(x, v^{\prime}, t\right) d v^{\prime} d v d x-\int \sigma\left(x_{D} v\right) n(x, v, t) d v d \\
& =\int\left(\sigma_{\varrho}(x, v)-\sigma_{u}(x, v)\right) n(x, v, t) d x d v \\
& \leqq-\alpha\|n(t)\|_{D} .
\end{aligned}
$$

Choose $T$ so large (by Lemma 4.2) that for $t>T, n(x, v, t) \neq 0$ if $x \in S_{r} \backslash D, x \notin C_{x}$. Then, by Lemma 4.3 , the term

$$
\int\left(v \cdot \operatorname{grad}_{x} p\right) \frac{\partial \phi}{\partial y} n(x, v) d x d v \leqq 0
$$

since supp $\frac{\partial \phi}{\partial y} \subset S_{r} \backslash D$ and $\frac{\partial \phi}{\partial y} \leqq 0$ by monotonicity. It follows that

so for $t \geqq t_{0} \geqq T$

$$
\frac{d}{d t}\|n(t)\|_{\phi} \leqq\|n(t)\|_{D}, \quad t \geqq T
$$

$$
\|n(t)\|_{\phi} \leqq\left\|n\left(t_{0}\right)\right\|_{\phi}-\alpha \int_{t_{0}}^{t}\|n(s)\|_{D} d s .
$$

As supp $\phi$ approaches $\{0\},\|n(t)\|_{\phi} \rightarrow\|n(t)\|_{D}$ so we have:

$$
\|n(t)\|_{D} \leqq\left\|n\left(t_{0}\right)\right\|_{D}-\alpha \int_{t_{0}}^{t}\|n(s)\|_{D} d s .
$$

From (16) we conclude that $\|n(t)\|_{0}$ is monotone decreasing for $t>T$, and from this that for any $\delta$

$$
\left\|n\left(t_{0}+\delta\right)\right\|_{D} \leqq(1-\alpha \delta)^{-1}\left\|n\left(t_{0}\right)\right\|_{D} .
$$

Taking $\delta=\left(t-t_{0}\right) / n$ and letting $n \rightarrow \infty$. we have:

$$
\|n(t)\|_{D} \leqq e^{-\alpha\left(t-t_{0}\right)}\left\|n\left(t_{0}\right)\right\|_{D} .
$$

Proof of Theorem 4.1. From Theorem 4.4, for any $n \in \hat{C}_{0}^{\infty}, \int_{0}^{\infty}\|W(t) n\|_{D} d t<\infty$. Thus, by Theorem 3.1, $\tilde{\Omega}^{-}$exists.

\section{Appendix. An Example with Non-Invertible Dynamics}

Let $D$ be the cube $(-1,1)^{3}$ let $k\left(x, v, v^{\prime}\right)=v v^{\prime} \exp \left(-v^{2}-v^{\prime 2}\right) ; x \in D$ and $\sigma_{\alpha}(x, v)=\sigma_{\varrho}(x, v)$ and let $n$ be a positive $L^{1}$ function whose $x$-space support is in a very small neighborhood of $\left\langle 1+10^{-6}, 0,0\right\rangle$ and whose $v$-space support is in 
a cone of very small opening angle about $\langle 1,0,0\rangle$ with $1 \leqq|v| \leqq 2$. Then we claim that $n$ is not in the range of $W(t)$ for say $t \geqq 1$ as an operator on $L_{+}^{1}$ (so in particular the inverse of $W(1)$ on $L^{1}$ is not positivity preserving). To prove our claim we note that for particles to be in supp $n$ at $t=1$, they must have passed through $D$ and thus they have scattered with nonzero probability. Thus the distribution for $v$ must have a Gaussian piece and, in particular, cannot have $v$-space support strictly in $\{v|1 \leqq| v \mid \leqq 2\}$.

\section{References}

1. Bell, I., Glasstone, S. : Nuclear reactor theory. Princeton, New Jersey: Van Nostrand 1970

2. Chernoff, P.: Note on product formulas for operator semigroups. J. Funct. Anal. $2238-242$ (1968)

3. Cohen,E., Thirring, W.: The Boltzmann equation. Berlin-Heidelberg-New York: Springer 1973

4. Cook, J.: Convergence to the Mølers wave matrix. J. Math. Phys. 36, 82-87 (1957)

5. Davison, B., Sykes, B.: Neutron transport theory. Oxford: Oxford University Press 1958

6. Ford, G., Uhlenbeck, G.: Lectures in statistical mechanics, A. M.S. Publications

7. Hejtmanek, J.: Streutheorie des Linearen Boltzmannoperators, Univ. of Wien Preprint

8. Hille,E., Phillips, R.S.: Functional analysis and semigroups. A. M.S. Colloq. Publ. 31, 1957

9. Jörgens, K.: An asymptotic expansion in the theory of neutron transport. Comm. Pure Appl. Math. 11, 219-242 (1958)

10. Kato, T.: Perturbation theory for linear operators, Berlin-Heidelberg-New York: Springer 1966

11. Kato, T.: Wave operators and similarity for non-self adjoint operators. Math. Ann. 162, 258-279 (1966)

12. Lavitz, J., Marchand, J. P.: ed. Mathematical theory of scattering. Amsterdam: North Holland 1974

13. Lax, P., Phillips, R.: Scattering theory for dissipative hyperbolic systems. J. Funct. Anal. 14, $172-235$ (1973)

14. Lehner, J., Wing, G.: On the spectrum of an unsymmetric operator arising in the transport theory of neutrons. Comm Pure Appl. Math 8, 217-234 (1955)

15. Lehner, J., Wing, G.: Solution of the linearized Boltzmann equation for the slab geometry. Duke Math. J. 23, 125-142 (1956)

16. Mihalas, D.: Stellar atmospheres. W. H. Freeman \& Co. 1970

17. Nelson, E.: Analytic vectors. Ann. Math. 10, 572-614 (1959)

18. Reed,M., Simon, B.: Methods of modern mathematical physics, Vol. II Fourier Analysis, SelfAdjointness, New York: Academic Press, in press, expected May, 1975

19. Reed, M., Simon, B.: Methods of Modern Mathematical Physics, Vol. III, in preparation, expected publication Summer 1976

20. Trotter, H.: On the product of semigroups of operators. Proc. A. M.S. 10, 545-557 (1959)

Communicated by W. Hunziker
B. Simon

Departments of Mathematics and Physics

Princeton University

Princeton, New Jersey 08540, USA 